\title{
Intravascular ultrasound, performed during resuscitative endovascular balloon occlusion of the aorta (REBOA), confirms correct balloon deployment and haemostasis - a potential solution for remote, austere and military settings
}

\author{
P S C Rees, A M Buckley, S A Watts, E Kirkman
}

\begin{abstract}
Introduction

Resuscitative endovascular balloon occlusion of the aorta (REBOA) is rapidly evolving as an emergency haemorrhage control technique. It has wide potential applicability in remote and austere settings, and following military trauma where prolonged field care might be required. However, rapid confirmation of balloon delivery is a challenge which relies on estimates derived from anatomical measurements or trans-abdominal ultrasound. In addition, confirmation of adequate balloon expansion is difficult. Intravascular ultrasound (IVUS) offers a solution to these two issues, making REBOA a deliverable therapy in the pre-hospital and early hospital settings.
\end{abstract}

\section{Methods}

In an animal model of severe ballistic trauma, following characterisation of the technique, an IVUS-REBOA device was configured, combining a peripheral angioplasty balloon and a digital coronary IVUS catheter. This was introduced via a sheath into the femoral vessel over a conventional angioplasty guide wire.

\section{Results}

Real time IVUS imaging allowed confirmation of delivery of the balloon to the aorta, and also demonstrated full apposition once deployed. Furthermore, using ChromaFlo imaging, the device confirmed loss of pulsatile flow in the aorta after deployment, correlating with loss of transduced femoral pressure traces. Post-mortem examination confirmed correct anatomical balloon placement.

\section{Summary}

For the first time, in a porcine pilot study, we have demonstrated that IVUS-REBOA is feasible and confirms both correct balloon placement and haemostasis. It has potential to offer advantages to REBOA operators especially during the pre-hospital and retrieval phases, and in the early phase of hospital delivered damage control resuscitation at remote locations.

Rees P S C, Buckley A M, Watts S A, et al. J R Nav Med Serv 2018;104(1):12-17

\section{Introduction}

Haemorrhage is the primary cause of death following injuries sustained in conflict. Effective medical systems which deliver early resuscitation and control of bleeding are vital to preserve life. An analysis of 4,596 United States military deaths during kinetic operations in Iraq and Afghanistan demonstrated that $87.3 \%$ of deaths occurred early, prior to arrival at a medical treatment facility. $24.3 \%$ of deaths were assessed as potentially survivable had swifter intervention been possible. ${ }^{1}$

Non-compressible torso haemorrhage carries a high mortality, and presents a particular therapeutic challenge as it cannot easily be remedied by early medical intervention with pressure dressings, combat tourniquets or haemostatic trauma dressings. ${ }^{2}$ The only effective therapies for shocked patients in this group are early blood component transfusion, whilst preparing for emergency laparotomy or thoracotomy in order to gain proximal aortic haemorrhage control, followed by damage control surgery. If the patient presents in the peri-arrest phase, outwith a facility capable of providing immediate surgery, death is likely to ensue. Whilst the presence of a full military trauma system proved successful during the recent counter-insurgency operations in Afghanistan, ${ }^{3}$ this multi-professional system is difficult to mirror in less mature 
contingency operations. Often assembled at relatively short notice, casualty evacuation may be hampered by an inability to allow air, boat or ground transport assets to be dedicated solely for medical transport. Similarly, casualty evacuation timelines may be long due to geographical distance, unfavourable meteorological variables or tactical issues. Accordingly, a haemorrhage control solution that could be deployed into a prolonged field care or early in-hospital setting is an attractive prospect with life-saving potential due to the ability to buy time for surgical haemorrhage control.

Resuscitative endovascular balloon occlusion of the aorta (REBOA) is a rapidly evolving solution to haemorrhage control. It involves occlusion of the descending aorta by delivery of a balloon catheter device via an introducer sheath placed in the common femoral artery - effectively acting as an endovascular aortic cross-clamp. Three anatomically-defined aortic landing zones are described: zone I - thoracic aorta between the left subclavian and coeliac artery; zone 2 - between the coeliac artery and renal arteries; zone 3 - between the renal arteries and aortic bifurcation. Only zones I and III are usually used as landing zones for emergency haemorrhage control. Commonly, balloon placement is performed under direct fluoroscopic guidance in the emergency department or in a hybrid interventional radiology or vascular surgical theatre. A small number of cases have been performed in the pre-hospital setting by clinicians of London's Air Ambulance, which deploys a helicopter-delivered, physician-led advanced trauma team to the scene of serious incidents. ${ }^{4}$

In multiple animal studies, REBOA has been shown to offer an effective endovascular solution for severe torso and pelvic haemorrhage, resulting in rapid control of bleeding and improved survival, most likely mediated by increased proximal blood pressure, brain oxygen delivery and carotid blood flow, compared with standard therapy ${ }^{5-7}$ In human cohorts, vascular surgeons have reported the use of balloon occlusion as an effective means of gaining rapid haemorrhage control in the setting of ruptured aortic aneurysm prior to endovascular repair, ${ }^{8,9}$ and a number of case series have been reported on the use of REBOA in the setting of major traumatic haemorrhage. To date, the medical literature contains data on 1355 cases treated with REBOA, including reports of non-trauma indications such as gastrointestinal and post-partum bleeding. ${ }^{10}$

Outside a resuscitation room equipped with fluoroscopy, with which X-ray screening can be used to track the passage of the balloon, confirmation of balloon delivery to the intended aortic landing zone presents a significant limitation to REBOA. A blind approach is possible, though the potential risk of device failure or unintentional balloon deployment in a smaller branch vessel exists. Trans-abdominal ultrasound techniques have been reported, using contrast-enhanced ultrasonography to confirm REBOA placement, but these require additional resources and training. ${ }^{11}$

Intravascular ultrasound (IVUS) is an endovascular imaging technique in common use in interventional cardiology and radiology. Using a miniaturised, solid-state imaging device deployed into the target vessel over a guidewire, it allows for real time two-dimensional imaging to be performed. The images obtained can be analysed to assess vessel diameter or the burden of atheroma, and to guide interventional therapies such as stents. IVUS devices are now small-calibre, simple to use and interpret, and are approved by international regulatory bodies for use in humans. In interventional cardiology, data strongly support IVUS use especially in complex cases, and international guidelines recommend their use. ${ }^{12} \mathrm{ChromaFlo}^{\mathrm{TM}}$ is an imaging modality which colourises movement in the IVUS image pixels as red, presenting a form of 2-dimensional Doppler. Pixel movement is thus used as a surrogate for flow, and this feature can be used to clearly delineate the vessel lumen. This study also aimed to evaluate its use in confirming loss of aortic flow following REBOA balloon deployment, as a potential tool for evaluating REBOA effect in vivo.

\section{Methods}

The study was ethically reviewed, approved, and conducted in accordance with the Animals (Scientific Procedures) Act 1986. Two large white swine, weighing $35 \mathrm{~kg}$, were terminally anaesthetised using isoflurane followed by alfaxalone. They were instrumented for central and cardiac blood pressure measurement, cardiac output measurement and the removal of blood and administration of medications and fluids. Following the completion of an experimental protocol simulating severe haemorrhagic shock followed by damage control resuscitation, the following pilot studies were performed:

\section{Run 1 - IVUS assessment}

To confirm the ability to gain access to and visualise the aorta, a 9 French gauge $(F)$ sheath with haemostatic valve and a side arm was placed in the right common femoral artery via a limited cut down to expose the vessel, using a modified Seldinger technique under direct vision. Through this, a $175 \mathrm{~cm}, 0.035$ " J-tipped guidewire was passed, over which a $10 \mathrm{MHz}, 8.5 \mathrm{~F}$ compatible digital IVUS catheter (Visions PV, Philips Volca$\mathrm{no}^{\mathrm{TM}}$ ) was introduced. The position of the aortic bifurcation was visualised under direct IVUS guidance, and the diameter of the distal aorta was noted to aid REBOA balloon selection for further runs. The IVUS catheter was then removed.

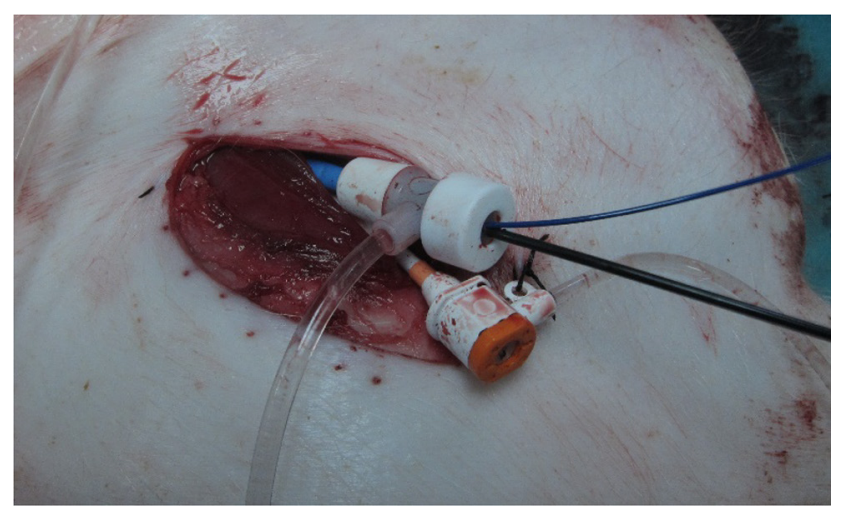

Figure 1: Right femoral access site. Limited cut-down, with $12 \mathrm{~F}$ sheath in femoral artery. REBOA balloon catheter shaft (right of picture) and IVUS catheter passing through haemostatic valve. A venous sheath is also present. 


\section{Run 2 - ChromaFlo}

Through the femoral sheath, a 0.014" angioplasty guidewire (Balance Middle Weight, Abbott ${ }^{\mathrm{TM}}$ ) was introduced into the aorta. A hydrophilic-coated $20 \mathrm{MHz}, 5 \mathrm{~F}$ compatible digital

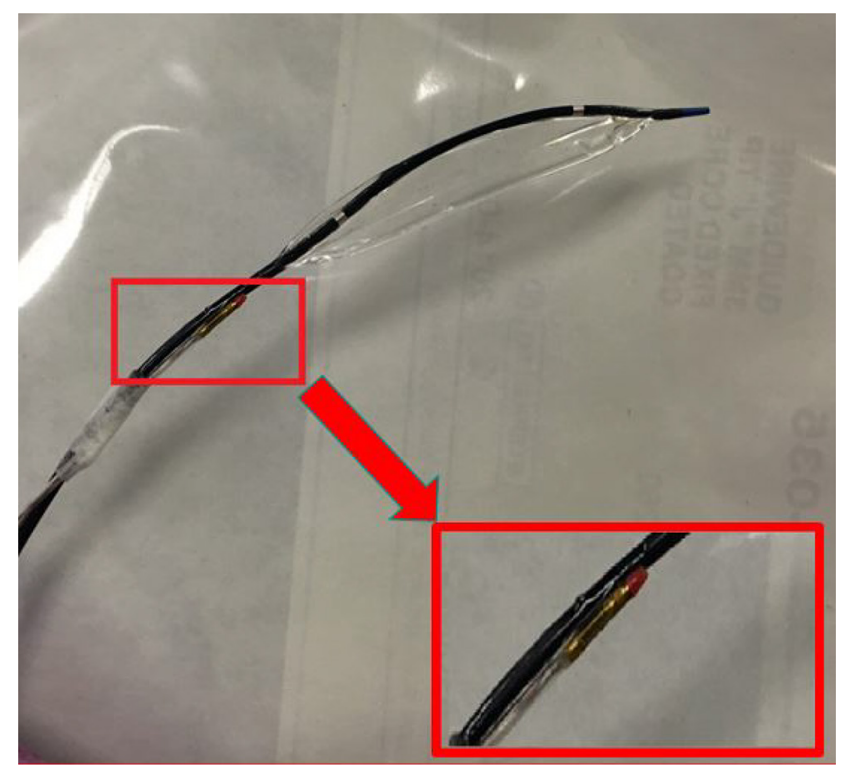

Figure 2: - Tip of combined IVUS-REBOA balloon catheter, with balloon deflated. Inset: close-up of IVUS catheter attached to balloon catheter shaft (Eagle Eye Platinum, Philips Volcano ${ }^{\mathrm{TM}}$ ).

IVUS catheter was passed over this (Eagle Eye Platinum, Philips Volcano ${ }^{\mathrm{TM}}$ ). Once delivered, ChromaFlo imaging was commenced and the image was interpreted.

\section{Run 3 - ChromaFlo guided REBOA}

With the IVUS catheter in situ in the distal aorta, a $9.0 * 40 \mathrm{~mm}$ angioplasty balloon (Mustang, Boston Scientific ${ }^{\text {TM}}$ ) was passed over a 0.035 " guidewire. The balloon was deployed, and the aortic flow was monitored using both ChromaFlo and observation of the transduced pressure trace from

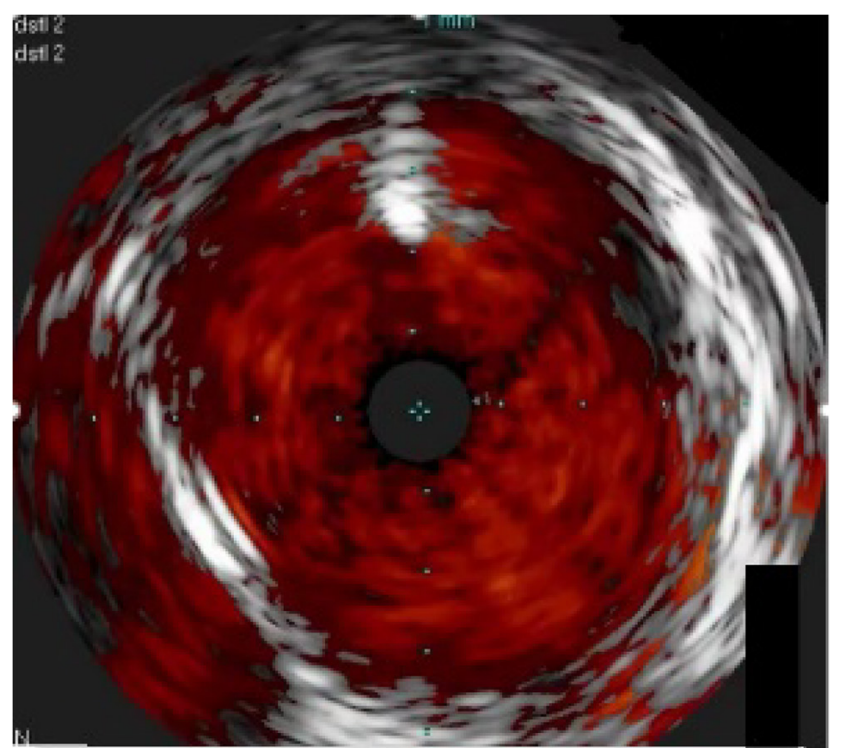

Figure 3: Representative aortic IVUS image. ChromaFlo is activated, delineating areas of blood flow. A 0.035" guidewire is noted at the top of the image as a bright signal, and the inferior vena cava is also captured to the left of the aortic signal.

the contralateral femoral arterial sheath (LabChart, ADI Instruments $^{\mathrm{TM}}$ ).

Run 4 - Combined IVUS-REBOA device

A bespoke IVUS-REBOA device was fashioned by combining the angioplasty balloon and the IVUS probe. Multiple REBOA runs were performed, delivering the device through the right femoral artery sheath to the distal aorta with ChromaFlo active. Once in position, the balloon was deployed, and the clinical effect was assessed using both ChromaFlo and observation of the transduced pressure trace from the contralateral femoral arterial sheath.

The position of the balloon from the final run was confirmed by direct visualisation of the opened aorta at post-mortem examination.

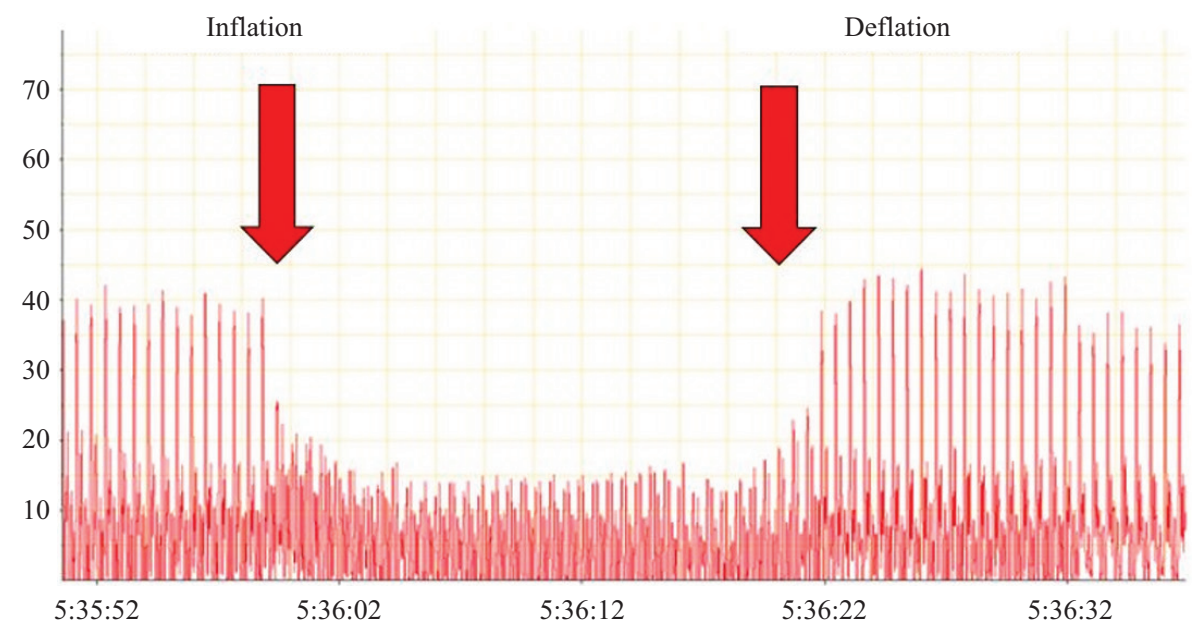

Figure 4: Transduced femoral arterial trace during IVUS-REBOA deployment and deflation. A marked reduction in downstream pressure is visible during balloon deployment, which resolves on balloon deflation. 


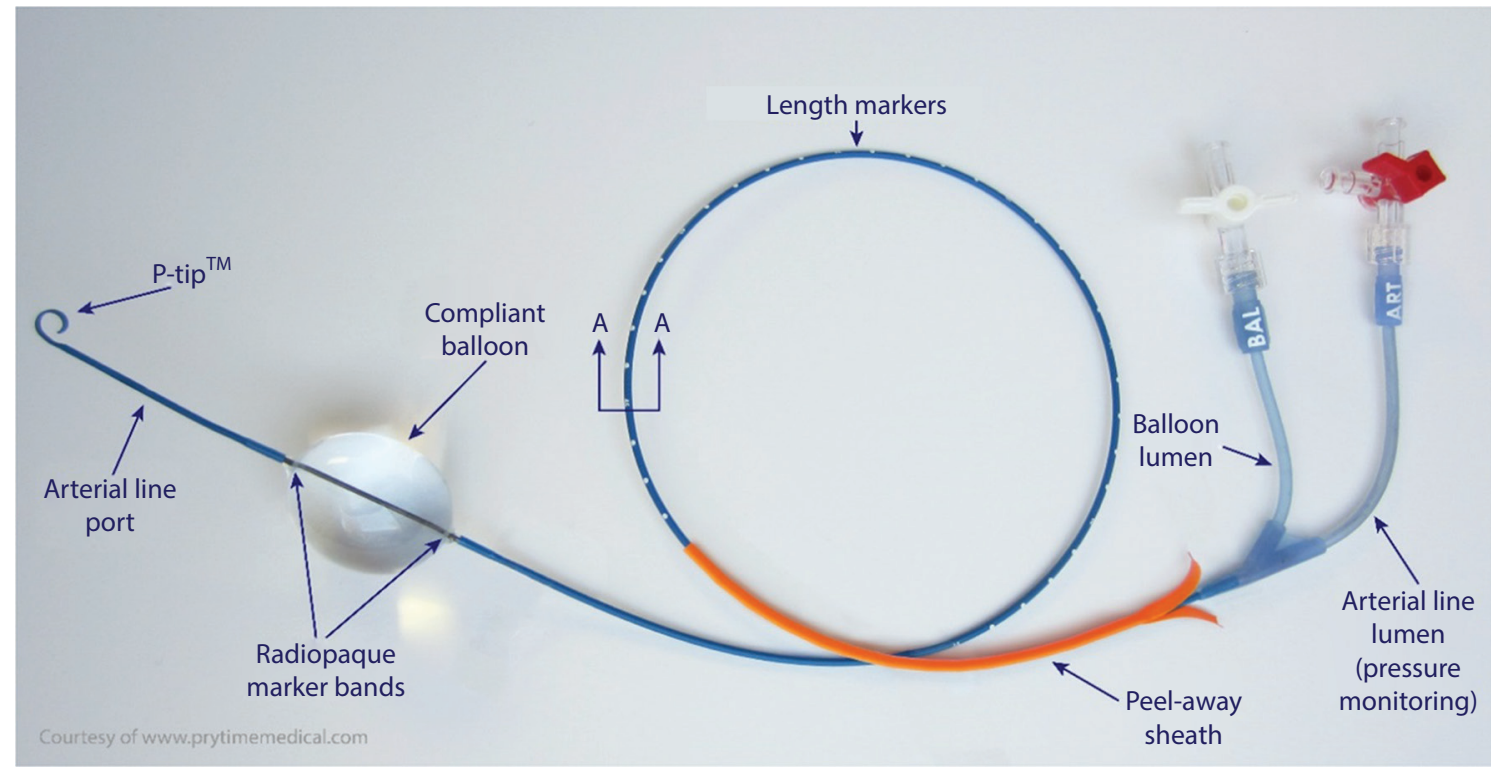

Figure 5: Commercially available REBOA device (ER-REBOA ${ }^{\mathrm{TM}}$ catheter, image courtesy of Prytime Medical) for comparison, with balloon deployed.

\section{Results}

Two pigs were studied in this initial technical evaluation pilot. The results of each component are reported. Femoral access was gained bilaterally without incident in both subjects.

Run 1 - delivery of the 0.035 " guidewire and larger Vision catheter was successful at first pass in both cases, and internal diameter of the target aortic area was noted as $\sim 10 \mathrm{~mm}$.

Run 2 - delivery of the angioplasty guidewire and Eagle Eye catheter was successful at first pass in both cases. ChromaFlow imaging clearly delineated the aortic wall, and confirmed pulsatile flow in time with the cardiac cycle and corresponding to the transduced arterial pressure traces. Even in the setting of cardiac arrest with chest compressions underway (according to study protocol) by a mechanical cardiopulmonary resuscitation device (LUCAS 2, Physio Control ${ }^{\mathrm{TM}}$ ), ChromaFlo was able to detect the presence of aortic flow.

Run 3 - delivery of the REBOA guidewire and balloon was successful at first pass, and easily visualised using live IVUS imaging, with both guidewire and balloon shafts visible as they passed the IVUS catheter. In addition, on deployment of the REBOA balloon, ChromaFlo pulsation was lost simultaneously with a dramatic reduction in transduced distal arterial pressure trace.

Run 4 - delivery of the IVUS-REBOA device was successful at first pass in all cases (total 10 runs). IVUS imaging confirmed correct deployment of the device above the iliac bifurcation, in aortic zone 3. ChromaFlo imaging confirmed full apposition of the REBOA balloon to the aortic wall, and loss of pulsatile signal confirmed aortic occlusion, again corresponding with loss of transduced distal arterial pressure trace. On deflating the REBOA balloon, forward flow rapidly re-established, confirmed by restoration of a visible pulsatile
ChromaFlo signal and transduced distal arterial pressure trace. The mean time taken from balloon insertion into the femoral sheath to balloon deployment was 21.25 seconds ( $n=10$ runs, range 18.1-24.0 seconds).

Post-mortem examination confirmed correct delivery of the IVUS-REBOA device to the distal aortic landing zone in both subjects. No vascular injuries related to either vascular access or REBOA were found.

\section{Discussion}

This series of studies demonstrates, for the first time in a porcine model, that a commercially available IVUS catheter can be used to confirm delivery of the REBOA balloon to the correct anatomical landing area. It also allows real-time observation of balloon deployment and confirms apposition of the balloon to the vessel wall. The addition of ChromaFlo imaging allowed confirmation of loss of pulsatile aortic flow distal to the deployed balloon, confirming clinical effect. Deployment of the device was rapid, safe and did not require fluoroscopy.

\section{Limitations}

This was a proof-of-concept pilot study, hence the number of animal subjects was small. An expanded study using a novel pressurised human cadaveric model is planned. The femoral access devices used were relatively large (9-12F) due to the calibre required to introduce the IVUS catheter as a piggy-backed device coupled to the REBOA balloon. If a combined device were to be manufactured, with a lower cross-sectional profile, a smaller femoral sheath could be used. A recent multicentre registry study confirms that the use of smaller sheaths is associated with fewer access site complications, such as thrombus and distal ischaemia. ${ }^{13}$ The use of smaller sheaths (e.g. 7F) is likely to broaden REBOA delivery among physicians who are not regular endovascular operators. In 
addition, the use of vascular access sites other than the femoral area is attractive, especially following trauma where the groin might be anatomically disrupted and involved in the primary injury. Interventional cardiology procedures, using catheters most commonly $6 \mathrm{~F}$, but ranging from $5-7.5 \mathrm{~F}$, predominantly use the radial artery as the primary access site of choice, due to lower procedural access site related complications. REBOA from non-femoral sites has been reported, but confirmation of delivery to the intended target landing zone would usually mandate fluoroscopy. Forward-looking, three-dimensional IVUS, currently an investigative tool, might offer a solution to this, allowing the operator to steer the aortic balloon from the subclavian system into the descending aorta, and on to the intended landing zone. Staying away from the femoral site would also reduce procedural complications such as pseudoaneurysm formation, the development of arteriovenous fistulae and post-procedural bleeding. Care of the radial access site is also much easier, using direct pressure with a currently available compression band device (e.g. TR band, Terumo ${ }^{\mathrm{TM}}$ ).

IVUS imaging requires connection of the endovascular part of the catheter to an external processing unit via a connector cable. This is currently built solely for in-hospital use in the interventional suite, and is a wheeled tower containing a processing unit and a display screen. Whilst the current unit could be suitable for emergency department-delivered IVUS-REBOA, it would require extensive miniaturisation to enable its use in the pre-hospital setting. Ultrasound devices using wireless probes such as the Accuson Freestyle ${ }^{\mathrm{TM}}$ have already been used by military units in austere settings, ${ }^{14}$ and the development of a combined IVUS-REBOA device which connects wirelessly to a handheld tablet-sized screen could easily be achieved and make this a therapy which is truly suited to pre-hospital and prolonged field care situations. Most critical care clinicians are used to interpreting ultrasound images, and little additional training uplift would be required to interpret IVUS images.

REBOA has the potential to save lives in a military trauma cohort. Retrospective analysis of a UK military cohort identified 174 combat deaths due to exsanguinating trauma, with a mean pre-hospital time of 61 minutes. $^{7} 66$ deaths occurred en route to hospital, and 29 shortly after arrival at the receiving facility. From injury analysis, $20 \%$ of patients had a focus of haemorrhage in the abdomen or around the abdomino-pelvic junction that could have been anatomically controlled with REBOA. It is likely that a significant number of the remainder could also have benefitted from the physiological stabilisation offered by REBOA. ${ }^{7}$ A population-based gap analysis of civilian trauma patients in England and Wales revealed similar data and suggested a role for REBOA mostly within high volume major trauma centres. ${ }^{15}$ REBOA is now well established as a potential therapy for exsanguinating trauma, which could significantly improve outcomes in critically ill patients for whom there are no viable options. REBOA, as part of an armoury of advanced endovascular techniques, may be ready for exploitation when planning deployed damage control resuscitation and surgery facilities for military operations. ${ }^{16}$ Potential UK military uses might include Role 2 units, especially where surgical capacity is limited to one operating table, and a potential requirement might exist to stabilise one patient whilst another undergoes damage control surgery. This would include the Royal Navy Role 2 Afloat, and Commando Forces Surgical Group Role 2 Light Manoeuvre units.

IVUS-REBOA could also help to mitigate downstream tissue ischaemia. With the REBOA balloon totally inflated, downstream haemorrhage stops. Unfortunately, this results in an accumulation of metabolites in downstream tissues. Long periods of balloon inflation have been shown to result in a rise in lactate, interleukin-6, an increased vasopressor requirement and respiratory distress syndrome. ${ }^{17}$ Partial REBOA (pREBOA), with subtotal aortic occlusion which allows a limited amount of forward flow, can be used to extend the therapeutic window as demonstrated in recent animal studies. pREBOA results in reduced levels of serum lactate, with reduced splanchnic injury, when compared with complete REBOA (cREBOA), and also avoids the supra-normal pressure surge seen in the vascular tree above the inflated REBOA balloon..$^{18}$ pREBOA thus offers a physiologically and haemodynamically tolerable option for endovascular haemorrhage control, which might extend the golden hour after traumatic injury. ${ }^{19}$ Pragmatically, an initial cREBOA inflation, to rapidly achieve control is advocated. Following this, pREBOA could be considered, supported by registry data which further suggests that pREBOA is associated with better outcomes in human subjects. ${ }^{13} \mathrm{Clin}$ ically, there is no easy way to confirm whether cREBOA or pREBOA has been achieved. IVUS imaging offers a solution to this, allowing direct visualisation of balloon apposition to the vessel wall, allowing the operator to see whether complete or partial occlusion has been performed. ChromaFlo imaging would allow the operator to visualise a degree of forward flow should pREBOA be desired. Further research is required to assess the optimum degree of forward flow, and it is likely that this will vary between patients. An endovascular variable aortic control (EVAC) system, regulating the degree of REBOA based on changes in proximal pressure has been trialled in an animal study, ${ }^{20}$ and IVUS-REBOA technology could have a role in developing future variants of such an EVAC catheter, able to automatically perform these functions.

IVUS could be part of an advanced endovascular resuscitation toolkit. As part of an advanced endovascular resuscitation strategy, REBOA offers a bridge to damage control surgery. If traumatic cardiac arrest ensues, access to the aorta gives the added advantage of allowing future therapies such as selective aortic arch perfusion using either oxygenated blood or novel resuscitation fluids..$^{21}$ If initial efforts still fail, the addition of a large-bore venous cannula would make full cardiopulmonary bypass possible following damage control surgery. This combination of techniques - emergency preservation and resuscitation (EPR) and resuscitation for cardiac arrest from trauma is currently undergoing a feasibility and safety study in the United States (EPR-Cat study; ClinicalTrials.gov identifier NCT01042015). It is likely, as data emerges, that REBOA, SAAP and EPR will overlap to form a hybrid advanced endovascular toolkit for the management of haemorrhagic shock. ${ }^{22}$ Developing a robust REBOA capability is the first step to enabling deployed damage control resuscitation teams to be able to exploit these techniques as the data emerges in future. 


\section{References}

1. Eastridge BJ, Mabry RL, Seguin P. Death on the battlefield (2001-2011): implications for the future of combat casualty care. $J$ Trauma Acute Care Surg 2012;73:431-7.

2. Morrison JJ, America TER. Noncompressible torso hemorrhage: a review with contemporary definitions and management strategies. Surg Clin North Am 2012;92:843-58.

3. Penn-Barwell JG, Roberts, SAG, Midwinter, MJ. Improved survival in UK combat casualties from Iraq and Afghanistan: 2003-2012. $J$ Trauma Acute Care Surg 2015;78:1014-20.

4. Sadek S, Lockey DJ, Lendrum RA, Perkins Z, Price J, Davies GE. Resuscitative endovascular balloon occlusion of the aorta (REBOA) in the pre-hospital setting: an additional resuscitation option for uncontrolled catastrophic haemorrhage. Resuscitation 2016 Oct 31;107:135-8.

5. Avaro JP, Mardelle V, Roch A. Forty-minute endovascular aortic occlusion increases survival in an experimental model of uncontrolled hemorrhagic shock caused by abdominal trauma. J Trauma Acute Care Surg 2011;71:720-6.

6. Morrison JJ, Percival TJ, Markov NP. Aortic balloon occlusion is effective in controlling pelvic hemorrhage. J Surg Res 2012;177:341-7.

7. Morrison JJ, Ross JD, Houston RIV, Watson JDB. Use of resuscitative endovascular balloon occlusion of the aorta in a highly lethal model of noncompressible torso hemorrhage. Shock 2014;41:130-7.

8. Malina M, Veith F, Ivancev, K. Balloon occlusion of the aorta during endovascular repair of ruptured abdominal aortic aneurysm. $J$ Endovasc Ther 2005;12:556-9.

9. Matsuda H, Tanaka Y, Hino Y et al. Transbrachial arterial insertion of aortic occlusion balloon catheter in patients with shock from ruptured abdominal aortic aneurysm. J Vasc Surg 2003;38:1293-6.

10. Gamberini E, Coccolini F. Resuscitative Endovascular Balloon Occlusion of the Aorta in trauma: a systematic review of the literature. World J Emerg Surg 2017;12.

11. Chaudery M, Clark J, Morrison JJ, Wilson MH, Bew D, Darzi A. Can contrast-enhanced ultrasonography improve Zone III REBOA placement for prehospital care? J Trauma Acute Care Surg 2016;80:89-94.

12. Bavishi C, Sardar P, Chatterjee S et al. Intravascular ultrasound--guided vs angiography-guided drug-eluting stent implantation in complex coronary lesions: Meta-analysis of randomized trials. Am Heart J 2017;185:26-34.

13. Matsumura Y, Matsumoto J, Kondo H, Idoguchi JK. Fewer REBOA complications with smaller devices and partial occlusion: evidence from a multicentre registry in Japan. Emerg Med J 2017;34:793-9.

14. Rees PSC, Lamb LEM, Nicholson-Roberts TC et al. Safety and feasibility of a strategy of early central venous catheter insertion in a deployed UK military Ebola virus disease treatment unit. Intensive Care Med 2015;41:735-43.

15. Barnard EBG, Morrison JJ, Madureira RM. Resuscitative endovascular balloon occlusion of the aorta (REBOA): a population based gap analysis of trauma patients in England and Wales. Emerg Med J 2015;32:926-32.

16. Tisherman SA, Brenner ML. Taking advanced endovascular techniques out of the hospital: Ready for prime time. Resuscitation 2016;107:A3-A4.

17. Markov NP, Percival TJ, Morrison JJ, Ross JD, Scott DJ. Physiologic tolerance of descending thoracic aortic balloon occlusion in a swine model of hemorrhagic shock. Shock 2013;153:848-56.

18. Russo RM, Williams TK, Grayson JK. Extending the golden hour: partial resuscitative endovascular balloon occlusion of the aorta in a highly lethal swine liver injury model. J Trauma Acute Care Surg 2016;80:372-80.

19. Russo RM, Neff LP, Lamb CM. Partial resuscitative endovascular balloon occlusion of the aorta in swine model of hemorrhagic shock. $J$ Am Coll Surg 2016;223:359-68.

20. Williams TK, Neff LP, Johnson MA et al. Extending REBOA: endovascular variable aortic control (EVAC) in a lethal model of hemorrhagic shock. J Trauma Acute Care Surg 2016;81:294.

21. Manning JE, Murphy CA, Hertz CM. Selective aortic arch perfusion during cardiac arrest: a new resuscitation technique. Ann Emerg Med 1992;21:1058-65.

22. Kutcher ME, Forsythe RM, Tisherman SA. Emergency preservation and resuscitation for cardiac arrest from trauma. Int $J$ Surg 2016;33:209-12.

\section{Conflict of interest statement}

The authors report no conflicts of interest.

\section{Authors}

Surgeon Commander PSC Rees Royal Navy

Consultant in Interventional Cardiology, Acute and Pre-hospital Medicine

Reader in Military Medicine

Chair, Defence Resuscitation Committee

cardiacexpert@nhs.net

Academic Department of Military Medicine and University of St Andrews School of Medicine

Major AM Buckley RAMC

Academic Department of Military Medicine

Dr S A Watts, DSTL Porton Down

Dr E Kirkman, DSTL Porton Down 